\title{
Realizing High Thermoelectric Performance of Bi-Sb-Te-Based Printed Films through Grain Interface Modification by an In Situ- Grown $\beta-\mathrm{Cu}_{2-\delta}$ Se Phase
}

\author{
Md Mofasser Mallick,* Leonard Franke, Andres Georg Rösch, Sarfraz Ahmad, Holger Geßwein, \\ Yolita M. Eggeler, Magnus Rohde, and Uli Lemmer*
}

Cite This: https://doi.org/10.1021/acsami.1c13526

Read Online

ABSTRACT: It has been a substantial challenge to develop a printed thermoelectric (TE) material with a figure-of-merit $Z T>1$. In this work, high ZT p-type $\mathrm{Bi}_{0.5} \mathrm{Sb}_{1.5} \mathrm{Te}_{3}$-based printable $\mathrm{TE}$ materials have been advanced by interface modification of the TE grains with a nonstoichiometric $\beta-\mathrm{Cu}_{2-\delta} \mathrm{Se}$-based inorganic binder (IB) through a facile printing-sintering process. As a result, a very high TE power factor of $\sim 17.5 \mu \mathrm{W} \mathrm{cm}{ }^{-1} \mathrm{~K}^{-2}$ for a p-type printed material is attained in the optimized compounds at room temperature (RT). In addition, a high $Z T$ of $\sim 1.2$ at $\mathrm{RT}$ and of $\sim 1.55$ at $360 \mathrm{~K}$ is realized using thermal conductivity $(\kappa)$ of a pellet made of the prepared printable material containing 10

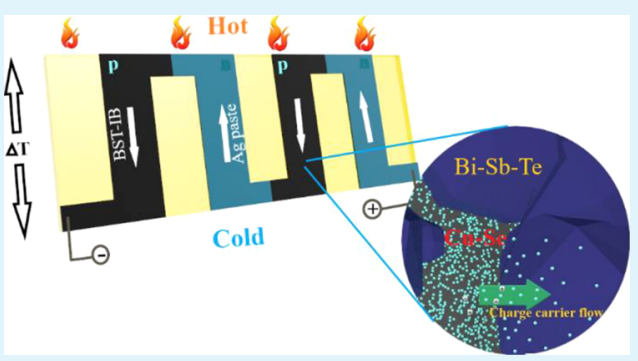
wt $\%$ of IB. Using the same material for p-type TE legs and silver paste for n-type TE legs, a printed TE generator (print-TEG) of four thermocouples has been fabricated for demonstration. An open-circuit voltage $\left(V_{\mathrm{OC}}\right)$ of $14 \mathrm{mV}$ and a maximum power output $\left(P_{\max }\right)$ of $1.7 \mu \mathrm{W}$ are achieved for $\Delta T=40 \mathrm{~K}$ for the print-TEG.

KEYWORDS: printed thermoelectrics, high ZT, Bi-Sb-Te alloy, $\mathrm{Cu}_{2} \mathrm{Se}$, printed TEGs

\section{INTRODUCTION}

Thermoelectrics is a simple technology with no moving component, which could be employed to recover waste heat into electricity. Approximately $65 \%$ of used energy is being wasted as heat; hence, converting waste heat into electricity via thermoelectric generators (TEGs) can significantly boost the renewable energy sector. However, several roadblocks hold researchers back from reaching the goal. ${ }^{1}$ So far, the vast majority of work on thermoelectrics has been involved in designing bulk TE materials and improving their figure-ofmerit $\left(Z T=S^{2} \sigma T / \kappa\right.$, where $S, \sigma$, and $\kappa$ are the Seebeck coefficient, electrical conductivity, and thermal conductivity), ${ }^{2-8}$ but lesser effort has been put forward to develop costeffective TE devices. Even though a large number of highperformance TE materials with $Z T>1$ have been developed already, it is still a challenge to produce low-cost and highoutput power-density TE devices using bulk materials because of a complex manufacturing process. ${ }^{9}$ Furthermore, the TE devices made of bulk materials are generally found in cuboids or other symmetric shapes. Hence, they are not easy to be integrated into nonflat surfaces of different systems. Also, the conventional TEGs with low internal resistance encounter problems when the external circuit's contact resistance is higher. As a result, the overall performance of the TEG is found to be diminished. On the other hand, printing technologies can be implemented in thermoelectrics to overcome the limitations associated with conventional TE devices. ${ }^{10}$ High-performance printable TE materials could offer low production cost, shape-versatility, rapid manufacturing, and high-output-power density devices for large-scale applications. ${ }^{11,12}$ However, printed materials with good printability and $Z T>1$ are difficult to design. In particular, the TE parameters $\sigma$ of inorganic-based printed materials are affected during ink processing, resulting in low ZT. Organicbased materials with good printability are there, but they exhibit low performance mainly because of very low $S .^{13-17}$ Also, to fabricate an efficient printed TE device, both n- and $\mathrm{p}$ type printed materials with similar performance are required. Among the conventional materials, $\mathrm{Bi}_{2} \mathrm{Te}_{3}$ and related compounds are commonly used for bulk TE devices. ${ }^{18,19}$ Hence, $\mathrm{Bi}_{2} \mathrm{Te}_{3}$-based $\mathrm{n}$ - and p-type bulk TE materials have been targeted extensively to make them printable, preserving their high performance at room temperature (RT). Unfortunately, the disruption of charge-carrier transport at the grain boundaries because of binders and additives lowers the overall $Z T{ }^{20} \mathrm{~A} Z T \sim 1$ was realized in a $\mathrm{Sb}_{2} \mathrm{Te}_{3}$-based printed film by connecting $\mathrm{Bi}_{0.4} \mathrm{Sb}_{1.6} \mathrm{Te}_{3}$ particles with melted $\mathrm{Te}$ particles.

Received: July 17, 2021

Accepted: November 4, 2021 
However, an intermediate postprinting high-pressure treatment is involved in processing. ${ }^{21}$ In a screen-printed $\mathrm{Bi}_{2} \mathrm{Te}_{3}$ film, a power factor value of $3.63 \mu \mathrm{W} \mathrm{cm} \mathrm{cm}^{-1} \mathrm{~K}^{-2}$ with $Z T \sim 0.2$ was attained through interface modification. ${ }^{22}$ In inkjet-printed $\mathrm{Bi}_{2} \mathrm{Te}_{3}$ and $\mathrm{Bi}_{0.5} \mathrm{Sb}_{15} \mathrm{Te}_{3}$ flexible nanowire films, power factors of $1.80 \mu \mathrm{W} \mathrm{cm} \mathrm{Cm}^{-1} \mathrm{~K}^{-2}$ and $1.10 \mu \mathrm{W} \mathrm{cm} \mathrm{cm}^{-1} \mathrm{~K}^{-2}$ were achieved through postprinting thermal annealing, respectively. ${ }^{23}$ In other reports, ${ }^{24-26} \mathrm{ZT}$ values of 0.3 and 0.2 are attained in pressure treatment-free p-type $\mathrm{Sb}_{2} \mathrm{Te}_{3}$-based films. $\mathrm{Bi}_{2} \mathrm{Te}_{3}$ based $\mathrm{p}$ - and $\mathrm{n}$-type painted/3D printed TE films with $Z T \sim 1$ were also reported using $\mathrm{Sb}_{2} \mathrm{Te}_{3}$ chalcogenidometallate as an inorganic binder (IB). The synthesis process is complicated and requires several steps. ${ }^{27-29}$ However, there is still no pressure treatment-free high-performance environmentally stable printable TE material reported for device applications because of either low $\sigma$ or poor printability. Hence, it has been a challenge to develop environmentally stable high-efficiency inorganic TE materials with good printability with a high power factor $\left(S^{2} \sigma\right)$ and low thermal conductivity $(\kappa)$.

In our previous work, we have developed a high-performance $\mathrm{Ag}_{2} \mathrm{Se}$-based n-type printable TE material ${ }^{30,31}$ using an organic binder. In this work, Bi-substituted $\mathrm{Sb}_{2} \mathrm{Te}_{3}$-based $\mathrm{p}$ type TE material $\mathrm{Bi}_{0.5} \mathrm{Sb}_{1.5} \mathrm{Te}_{3}$ has been targeted to make it printable, preserving its high RT TE performance using a $\mathrm{Cu}$ Se-based IB. For a $\mathrm{Bi}_{0.5} \mathrm{Sb}_{1.5} \mathrm{Te}_{3}$-based conventional printable ink containing $\mathrm{Bi}_{0.5} \mathrm{Sb}_{1.5} \mathrm{Te}_{3}$ particles, an organic binder, an additive, and a solvent, the electrical conductivity $\sigma$ of a sintered-printed film is found to be very low because of interruption of the electronic transport at the highly resistive grain boundaries, which leads to low $Z T$. Here, we report a high-performance $\mathrm{Bi}_{0.5} \mathrm{Sb}_{1.5} \mathrm{Te}_{3}$-based p-type printed material with $Z T>1$ using a $\mathrm{Cu}$-Se-based IB without a mechanical pressure treatment. Our work is inspired by the enhanced TE performance observed for a combination of a bulk half-Heusler alloy, $\mathrm{HfCoSb}$, and a band-matched chalcogenide, $\mathrm{Cu}_{2} \mathrm{Te}$ with the same charge carrier polarity, at the interfaces ${ }^{32}$ leading to a phonon filtering effect. Similarly, the reason behind using the $\mathrm{Cu}$-Se-based IB instead of previously reported n-type Ag-Sebased ink is that highly conducting bridges of the p-type $\beta$ $\mathrm{Cu}_{2-\delta} \mathrm{Se}$ phase are formed through postprinting sintering via dissociative adsorption of $\mathrm{Se}$ by $\mathrm{Cu}$. As a result, the p-type $\mathrm{Bi}_{0.5} \mathrm{Sb}_{1.5} \mathrm{Te}_{3}$ grains are bridged by a similar-charge carrier polarity nonstoichiometric p-type $\beta$ - $\mathrm{Cu}_{2-\delta}$ Se phase preserving their high electrical conductivity and high $Z T$.

\section{EXPERIMENTAL SECTION}

2.1. Materials. Ingots of p-type $\mathrm{Bi}_{0.5} \mathrm{Sb}_{1.5} \mathrm{Te}_{3}$ (beads, $99.99 \%$ trace metals basis, Sigma-Aldrich), copper powder (spheroidal) (10-25 $\mu \mathrm{m}, 98 \%$, Sigma-Aldrich), Se powder (100 mesh, $\geq 99.5 \%$ trace metals basis, Sigma-Aldrich), N-methyl-2-pyrrolidone (NMP) (anhydrous, $99.5 \%$, Sigma-Aldrich), polyvinylpyrrolidone (PVP) (average $M_{\mathrm{w}}$ $\sim 40,000$, Sigma-Aldrich), fumed silica (Evonik), and Kapton substrate (DuPont de Nemours) were used.

2.2. Experimental Methods. 2.2.1. Ink Formulation. To prepare a $\mathrm{Cu}$-Se-based IB, the powder elements $(\mathrm{Cu}$ and $\mathrm{Se})$ were weighed in a stoichiometric ratio. The powder elements were then mixed with an $\mathrm{NMP} / \mathrm{PVP}$ solution (organic solvents NMP/binder $(\mathrm{PVP})=92: 8$ ) in a 3:2 ratio followed by adding excess $0.5 \mathrm{wt} \%$ fumed silica. The obtained mixture was stirred for $>4 \mathrm{~h}$ at $1000 \mathrm{rpm}$. The p-type $\mathrm{Bi}_{0.5} \mathrm{Sb}_{1.5} \mathrm{Te}_{3}$ (BST) was ground to powder using a mortar and pestle for $20 \mathrm{~min}$. The resulting powder was then mixed with the $\mathrm{Cu}$-Sebased IB in different ratios, $(1-x) \mathrm{BST}-(x) \mathrm{IB}, x=0$ to 1 to optimize their performance by characterizing their morphological and physical properties. " $x$ " is the weight fraction (weight percentage, wt $\%$ of IB = $x \times 100)$ of IB in the BST-IB blend. It should be noted that the IB should be placed on a magnetic stirrer with low speed while collecting it using a syringe to avoid agglomeration and inhomogenization. Then, an additional $\sim 20 \mathrm{wt} \%$ of the NMP/PVP solution is added to the mixture. The final mixture was then stirred for $2 \mathrm{~h}$ at $1000 \mathrm{rpm}$ at $\mathrm{RT}$. The synthesis methods of the BST-IB-based inks and printed TE materials are shown in Figure 1.

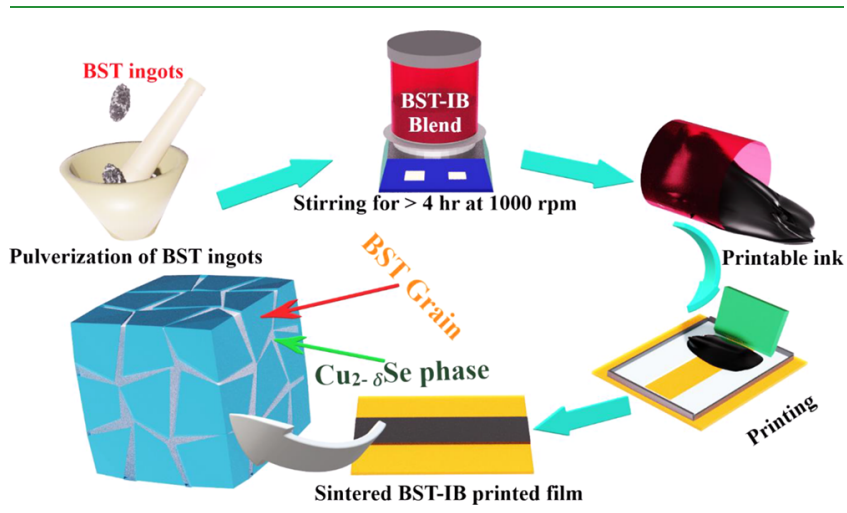

Figure 1. Routine of the synthesis processes of the BST-IB printed TE materials.

2.2.2. Film Preparation. The prepared inks were printed on different substrates (glass and Kapton) using the doctor-blading technique. The Kapton substrates were rubbed in one direction using emery paper before printing. Finally, the printed films with a thickness of $\sim 150-200 \mu \mathrm{m}$ were sintered at $703 \mathrm{~K}$ in a vacuum oven for 10 mins. Afterward, the samples were taken out after slow cooling down to below $350 \mathrm{~K}$. The density of the sintered films on glass substrates was estimated to be in the range between 2.33 and $2.5 \mathrm{~g} \mathrm{~cm}^{-3}$. A video clip of the printed films is given in Supporting Information, Video S1.

2.2.3. Characterization and TE measurements of the Films. The phase and crystal structure of the printed films were determined by $\mathrm{X}$ ray diffraction (XRD) on a Bruker D8 diffractometer with a Lynxeye XE detector and an Anton Paar HTK1200 heating chamber in BraggBrentano geometry using Ni-filtered $\mathrm{Cu} K \alpha_{1,2}$ lines. While the diffraction patterns were collected, a heating rate of $20 \mathrm{~K} \mathrm{~min}^{-1}$ with isothermal holds of $25 \mathrm{~min}$ at particular temperatures was used. The XRD patterns of the printed films were recorded varying the diffraction angle $(2 \theta)$ while the incident angle of the X-ray beam was fixed. The thicknesses and morphology of the printed films on glass substrates were measured using a Bruker 3D white-light interferometry (WLI) microscope. Backscattered electron (BSE) micrographs, secondary electron (SE) micrographs, and energy-dispersive X-ray spectroscopy (EDXS) analysis results were acquired using an FEI Quanta 650 environmental scanning electron microscope equipped with a Schottky field emitter and an SSD detector operated with 5 and $15 \mathrm{kV}$. The local-concentration line scans and the energy spectra were acquired and evaluated using Bruker EDXS Quantax 400 software. Temperature-dependent transport parameters, electrical conductivity $(\sigma)$, Hall coefficient $\left(R_{\mathrm{H}}\right)$, Hall mobility $\left(\mu_{\mathrm{H}}\right)$, carrier concentration $\left(p_{\mathrm{H}}\right)$, and type of charge carriers were determined using the Hall measurement technique (Linseis HCS 10) with a digital accuracy of $65 \mathrm{pV}$. Temperature-dependent $S$ of the printed films was measured using a custom-built setup with an experimental measurement error of $\sim 10 \%$ in the temperature range from 300 to $373 \mathrm{~K}$. The custom-built setup has been described in detail in the previous report. ${ }^{33}$ The measurement errors related to $\sigma$ and $\kappa$ are 6 and $10 \%$, respectively. Temperature-dependent bulk thermal conductivities $(\kappa)$ of $10 \mathrm{~mm}$ discs made of $(1-x)$ BST- $-(x) \mathrm{IB}$ inks by cold pressing at $50 \mathrm{MPa}$ pressure followed by sintering at $703 \mathrm{~K}$ in vacuum for $10 \mathrm{~min}$ were determined using a Netzsch LFA-427. The densities of the samples are $3.12,3.37,3.41,3.57$, and $4.58 \mathrm{~g} \mathrm{~cm}^{-3}$ for $x=0,0.05,0.1,0.2$, and 1 , respectively. 

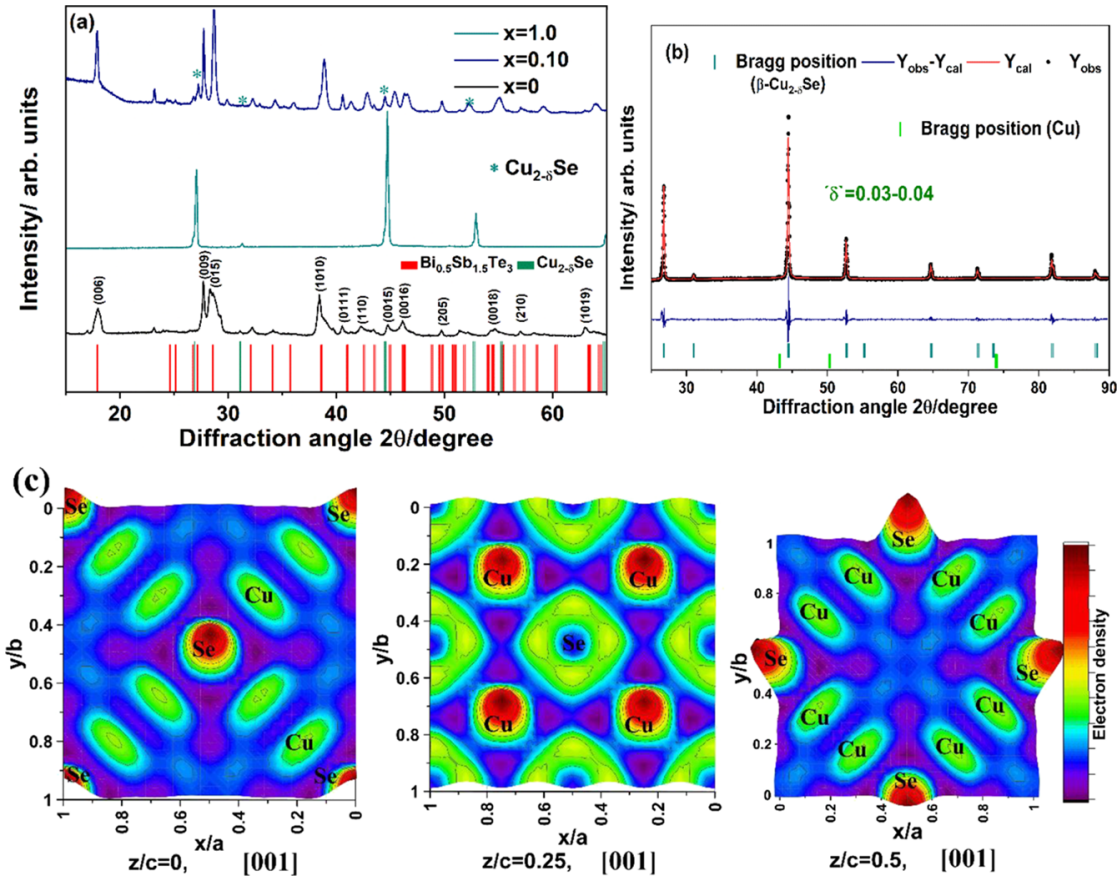

Figure 2. RT XRD patterns of the films $(1-x)$ BST $-(x)$ IB for $x=0, x=0.10$, and $x=1$ (a). The XRD pattern with Rietveld refinement results of the film with $x=1$ at RT (b). The cubic $\beta-\mathrm{Cu}_{2-\delta} \mathrm{Se}$ is found to be a prominent phase at RT. The Fourier maps at different planes along the [001] direction for electron density distributions of the atoms, $\mathrm{Cu}$ and $\mathrm{Se}$, in the $\beta$ - $\mathrm{Cu}_{2-\delta} \mathrm{Se}$ unit cell (c).

\section{RESULTS AND DISCUSSION}

\subsection{Crystallographic and Microstructure Analysis.} The crystallographic structures and phases of the printed films $(1-x) \mathrm{BST}-(x) \mathrm{IB}$ for $x=0, x=0.10$, and $x=1$ sintered at $703 \mathrm{~K}$ were investigated by XRD analyses at RT (c.f. Figure 2). The XRD pattern of the film with $x=0$ corresponds to the $\mathrm{p}$ type $\mathrm{Bi}_{0.5} \mathrm{Sb}_{1.5} \mathrm{Te}_{3}$ material with a trigonal crystal structure belonging to the space group $R \overline{3} m$ (PDF\#49-1713). The XRD pattern of the film for $x=0.10$ indicates the formation of the $\beta$ $\mathrm{Cu}_{2-\delta} \mathrm{Se}$ phase along with the main p-type $\mathrm{Bi}_{0.5} \mathrm{Sb}_{1.5} \mathrm{Te}_{3}$ phase. The RT XRD pattern of the pure IB film with $x=1$ indicates that the nonstoichiometric cubic $\beta-\mathrm{Cu}_{2-\delta} \mathrm{Se}$ phase is prominent together with a small amount of excess $\mathrm{Cu}$. To determine the $\mathrm{Cu}_{2-\delta} \mathrm{Se}$ phase formation temperature, temperature-dependent XRD analysis of the printed film with $x=1$ was performed from 298 to $673 \mathrm{~K}$ (cf. Figure S1). The film is found to be a mixture of unreacted $\mathrm{Cu}$ and $\mathrm{Se}$ at RT. The elemental $\mathrm{Cu}$ and Se start to react at a temperature $T>473 \mathrm{~K}$, and the $\mathrm{Cu}_{2} \mathrm{Se}$ phase is found to be formed at $623 \mathrm{~K}$ through thermally stimulated dissociative adsorption of Se by $\mathrm{Cu}$.

As a result, in all printed films with $0<x<1$, the BST TE grains are connected by the high-electrical conductivity $\mathrm{Cu}_{2} \mathrm{Se}$ phase minimizing the interfacial resistance at the grain boundaries. In addition, Figure 2a indicates that the printed films are polycrystalline. The lattices inside the grains of BST are mostly oriented in four different crystallographic planes including [006], [009], [015], and [1010]. To extract the indetail crystallographic structure of the $\mathrm{Cu}_{2} \mathrm{Se}$ phase at $\mathrm{RT}$, Rietveld refinement of the XRD pattern of the film with $x=1$ was performed using the FULLPROF program. The crystallographic structure with refinement results is shown in Figure $2 b$. The Rietveld refinement analysis indicates that the film corresponds to a primary nonstoichiometric cubic $\beta-\mathrm{Cu}_{2-\delta} \mathrm{Se}$ phase belonging to space group $F m \overline{3} m$ with a $<1$ vol \% of excess $\mathrm{Cu}$. The nonstoichiometry " $\delta$ " is found to be in the range of $0.04-0.05$. Typically, $\mathrm{Cu}_{2} \mathrm{Se}$ is found to be in the $\alpha$ $\mathrm{Cu}_{2} \mathrm{Se}$ phase with a complex structure at $\mathrm{RT}$, but nonstoichiometry leads to the cubic $\beta-\mathrm{Cu}_{2} \mathrm{Se}$ phase. It is reported that $\delta=0.03$ can cause a structural phase transition to the cubic $\beta-\mathrm{Cu}_{2} \mathrm{Se}$ phase. ${ }^{34}$ Hence, in our work, a cubic $\beta-\mathrm{Cu}_{2} \mathrm{Se}$ phase is formed because of nonstoichiometry " $\delta$ " in $\mathrm{Cu}_{2-\delta} \mathrm{Se}$. The lattice parameter of the $\beta-\mathrm{Cu}_{2-\delta} \mathrm{Se}$ phase unit cell is calculated to be $a=0.576(2) \mathrm{nm}$. The Se atoms in the $\beta$ $\mathrm{Cu}_{2-\delta} \mathrm{Se}$ crystal lattice are found to occupy Wyckoff position $4 \mathrm{a}$ $(0,0,0)$ in a face-centered cubic arrangement, and disordered $\mathrm{Cu}$ atoms are located in two different Wyckoff positions $8 \mathrm{c}(1 /$ $4,1 / 4,1 / 4)$ and $32 \mathrm{f}(x, x, x)$. Fourier maps for electron density distributions inside a unit cell of $\beta-\mathrm{Cu}_{2-\delta}$ Se at the planes $z / c=$ $0, z / c=0.25$, and $z / c=0.5$ along the direction [001] are shown in Figure 2c. It can be seen that the electron density distributions of the Se atoms at the $z / c=0$ plane are concentrated at the corners and the center $4 \mathrm{a}(0,0,0)$ position. Similarly, from the Fourier maps of the electron density distribution of atoms at $z / c=0.25$ and $z / c=0.5$ planes, the position of the $\mathrm{Cu}$ and $\mathrm{Se}$ atoms can be located to be $8 c(1 / 4,1 / 4,1 / 4)$ and $4 a(0,0,0)$. Overall, a homogeneous film is formed after printing and sintering. The morphology of a printed film with $x=0.1$ sintered at $703 \mathrm{~K}$ is analyzed using a white light interferometer which is found to be homogeneous, see Figure 3a. To understand the microstructures and interfacial effects, secondary electron micrographs, BSE micrographs, and EDXS analysis results of a polished cold pressed pellet made of the ink with $x=0.1$ were obtained (c.f. Figures $3 b, c$ and S2 and S3). The micrograph studies indicate a two-phase system, dark-spot $\mathrm{Cu}_{2-\delta} \mathrm{Se}$ phase embedded on the large $\mathrm{Bi}-\mathrm{Sb}-\mathrm{Te}$ grains. Line scan was performed across the interfaces of the two phases starting from a $\mathrm{Bi}-\mathrm{Sb}-\mathrm{Te}$ grain to observe the change in at $\%$ of the elements (c.f. Figure 3d). $\mathrm{The}$ at $\%$ of $\mathrm{Bi}, \mathrm{Sb}$, and $\mathrm{Te}$ elements starts to decrease, and prominently the at \% of the $\mathrm{Cu}$ starts to increase at the 

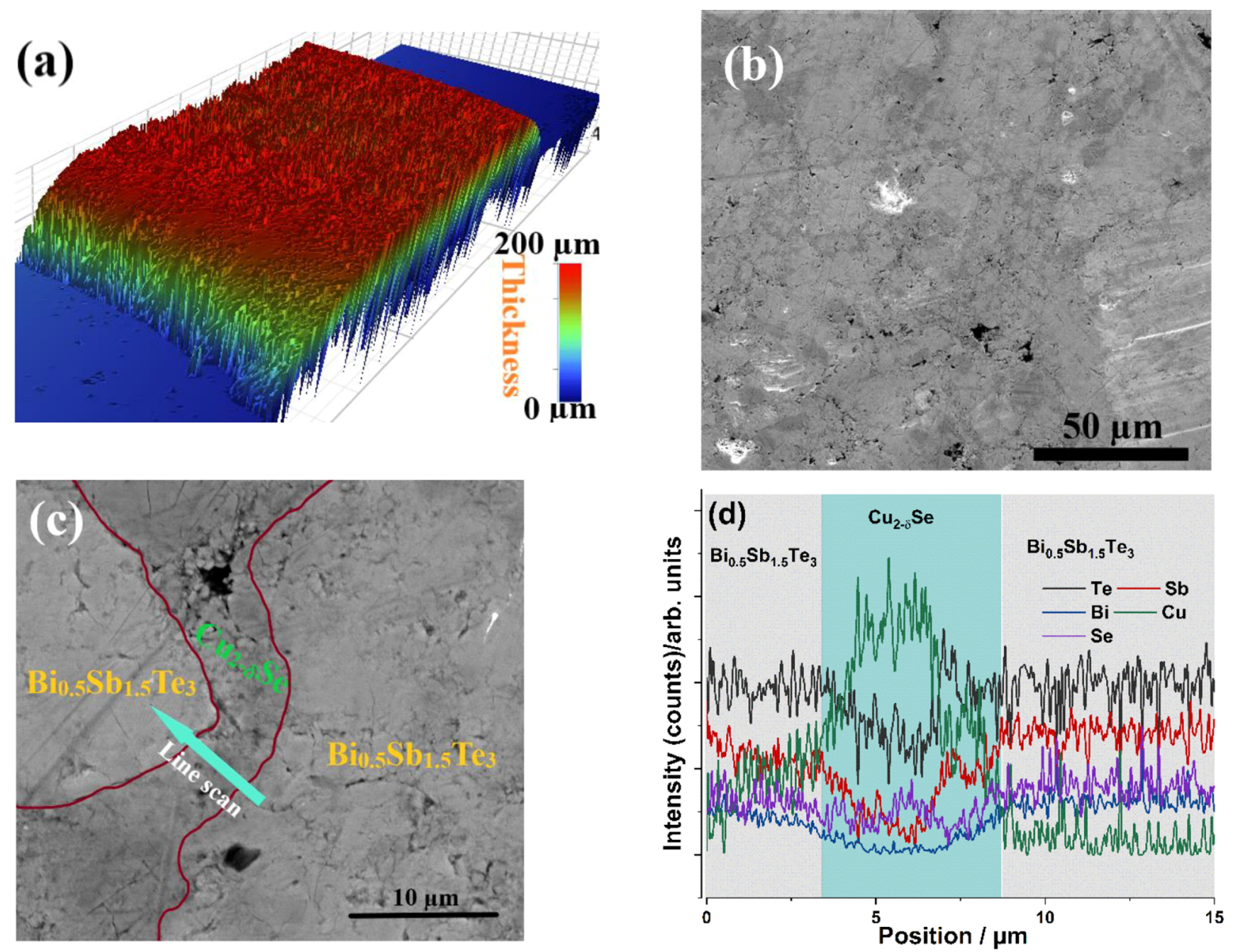

Figure 3. Morphology of the printed film for $x=0.10$ using WLI (a). Electron microscopy investigation of the $\mathrm{Bi}_{0.5} \mathrm{Sb}_{1.5} \mathrm{Te}_{-} \mathrm{Cu}_{2-\delta} \mathrm{Se}$ ink composite. Overview SE image acquired at $5 \mathrm{kV}$ exhibiting the topography of the ink after sintering (b). The micrograph reveals two types of material contrast (dark and bright gray regions). SE image at a higher magnification showing a close up of the two-phase region (c), and arrow indicates the location of the EDXS line scan revealed in (d).

interface between the $\mathrm{Bi}-\mathrm{Sb}$-Te grain and $\mathrm{Cu}_{2-\delta} \mathrm{Se}$ phase with higher charge carrier concentration. Thus, a modulationdoped-like two-phase system is manifested enhancing overall electrical conductivity. In elemental mapping of the printed film (Figure S4d), there is a clear contrast between the Bi-SbTe and $\mathrm{Cu}_{2-\delta} \mathrm{Se}$ phase. It should be noted that the EDS line profile only indicates in a nonquantitative manner the presence of the elements. The Se-signal shows an increase which, however, is obscured by the noisy Se-signal in the other regions. This can be explained by the volatile nature of the Se and the fact that we have performed the line scan in the backscattering mode. Thus, signals from the deeper layers of the samples could superimpose the signals from the surface.

\subsection{Thermoelectric (TE) Performance of Printed}

Films. First, the composition-dependent Seebeck coefficient of the printed films sintered at 623 and $703 \mathrm{~K}$ was studied, varying the $\mathrm{Cu}-\mathrm{Se}$-based inorganic binder IB wt \%. to identify the optimum composition and sintering temperature, Figure 4a. It is found that the films with $0.05 \leq x<0.2$ exhibit $S$ values of $>100 \mu \mathrm{V} \mathrm{K} \mathrm{K}^{-1}$ with a reasonably high electrical conductivity. Low $S$ for the printed films with $>20$ wt \% of IB indicates the formation of percolation paths of the $\mathrm{Cu}_{2-\delta} \mathrm{Se}$ phase, reducing the Seebeck coefficient of the material. According to 3D continuum models, the percolation threshold for a composite of two different lattices with a spherical shape is $\sim 29$ vol \% of any lattices. ${ }^{35,36}$ Above the percolation threshold, the electrical transport is dominated by the $\mathrm{Cu}_{2-\delta} \mathrm{Se}$ phase because of its higher electrical conductivity than the pristine BST film.

Hence, temperature-dependent thermotransport properties of the printed films with $\leq 20$ wt $\%$ of IB and the film with 100 wt $\%$ of IB on glass substrates were studied in the temperature range from 300 to $400 \mathrm{~K}$ in detail. The variation of Hall carrier concentration $\left(p_{\mathrm{H}}\right)$ and Hall carrier mobility $\left(\mu_{\mathrm{H}}\right)$ with temperature calculated from the Hall coefficient $\left(R_{\mathrm{H}}\right)$ is shown in Figure $4 b-d$. The RT transport parameters of the printed films are given in Table 1.

The $p_{\mathrm{H}}$ increases with increasing wt $\%$ of the IB in the films because of the higher content of higher carrier concentration $\mathrm{Cu}_{2-\delta} \mathrm{Se}$ phase. The $p_{\mathrm{H}}$ is found to be in the range between $2 \times$ $10^{19} \mathrm{~cm}^{-3}$ and $5 \times 10^{20} \mathrm{~cm}^{-3}$ for all the films. The variation of $p_{\mathrm{H}}$ with temperature is found to be insignificant for all films except for $x=0.20$ and $x=1.0$ at temperature $T>360 \mathrm{~K}$. On the other hand, the $\mu_{\mathrm{H}}$ is initially increased to $\sim 47 \mathrm{~cm}^{2} \mathrm{~V}^{-1} \mathrm{~s}^{-1}$ for $x=0.05$ and starts to decrease with increasing IB. For all the films except with $x=0$, the $\mu_{\mathrm{H}}$ values decrease with increasing temperature, and the change is more prominent for $x=0.20$ and $x=1.0$ at $T>360 \mathrm{~K}$ because of the creation of disordered $\mathrm{Cu}$ ions, which is consistent with the temperaturedependent $p_{\mathrm{H}}$. Inherently, BST alloys exhibit high carrier 

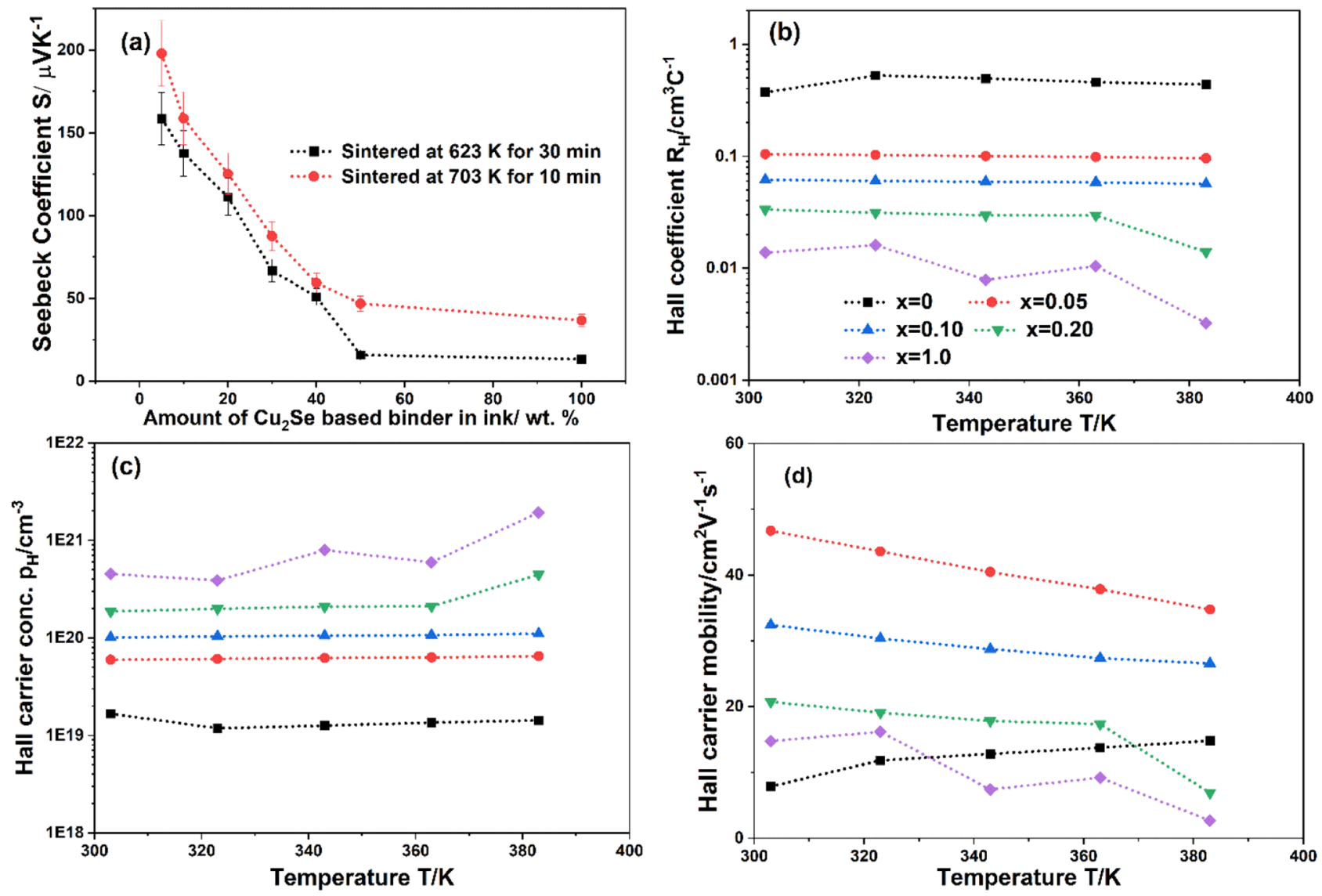

Figure 4. Composition-dependent Seebeck coefficients of the printed films sintered at 623 and $703 \mathrm{~K}$ (a). The Seebeck coefficient value is decreased with increasing Cu-Se-based IB. The temperature-dependent Hall coefficient $\left(R_{\mathrm{H}}\right)(\mathrm{b})$, Hall carrier concentration $\left(p_{\mathrm{H}}\right)(\mathrm{c})$, and Hall carrier mobility $\left(\mu_{\mathrm{H}}\right)(\mathrm{d})$ of the printed films $(1-x) \mathrm{BST}-(x)$ IB for $x=0$ to 1 .

Table 1. RT Transport Parameters, Hall Carrier Mobility $\left(\mu_{\mathrm{H}}\right)$, and Charge Carriers Concentration $\left(p_{\mathrm{H}}\right)$ Determined by Measuring the Hall Coefficient of Printed Films

\begin{tabular}{ccccc}
$\begin{array}{c}\text { electrical } \\
\text { films }\end{array}$ & $\begin{array}{c}\text { Hall } \\
\text { conductivity } \\
\sigma, \mathrm{S} \mathrm{cm} \mathrm{cm}^{-1}\end{array}$ & $\begin{array}{c}\text { Hall carrier } \\
\text { mobility } \\
\mu_{\mathrm{H}}, \mathrm{cm}^{2} / \mathrm{V} \mathrm{s}\end{array}$ & $\begin{array}{c}\text { Hall coefficient } \\
\text { concentration } p_{\mathrm{H},} \\
\times 10^{19} \mathrm{~cm}^{-3}\end{array}$ & $\begin{array}{c}R_{\mathrm{H},} \\
\times 10^{-2} \mathrm{~cm}^{3} / \mathrm{C}\end{array}$ \\
$\begin{array}{c}x=0 \\
x=\end{array}$ & 21 & 8 & 1.7 & 37.3 \\
0.05 & 447 & 47 & 6.0 & 10.4 \\
$\begin{array}{c}x= \\
0.10\end{array}$ & 524 & 32 & 10 & 6.2 \\
$\begin{array}{c}x= \\
0.20\end{array}$ & 617 & 21 & 18.6 & 3.3 \\
$\begin{array}{c}x= \\
1.0\end{array}$ & 1068 & 15 & 45.4 & 1.4 \\
\hline
\end{tabular}

mobility $>100 \mathrm{~cm}^{2} \mathrm{~V}^{-1} \mathrm{~s}^{-1}$, and the $\mathrm{Cu}_{2-\delta}$ Se shows low carrier mobility $<20 \mathrm{~cm}^{2} \mathrm{~V}^{-1} \mathrm{~s}^{-1}$. In this case, BST grains in the pristine printed film are not directly connected because of the presence of leftover binder, hence a high interfacial resistance, which suppresses the carrier mobility. The interfacial resistance is minimized enhancing resultant mobility by a low-resistive $\mathrm{Cu}_{2-\delta}$ Se path across the BST grain for the $x>0$ compositions. The temperature-dependent TE parameters in the range from 300 to $400 \mathrm{~K}$ of the films for $x=0$ to 1.0 are shown in Figure 5. The positive sign of the Seebeck coefficient $S$ for all the films indicates that the majority of charge carriers are p-type.

The pristine film with $x=0$ shows the highest $S$ value for all the temperatures with a maximum value of $\sim 240 \mu \mathrm{V} \mathrm{K}^{-1}$ at RT. The Seebeck coefficient $S$ increases with increasing temperature and reaches a maximum value at $\sim 360 \mathrm{~K}$ for all the BST-based films (c.f. Figure 5a). The pristine with $x=0$ film shows a semiconducting behavior with low conductivity. However, the electrical conductivity $\sigma$ is found to be much higher with values $>400 \mathrm{~S} \mathrm{~cm}^{-1}$ for the printed inorganic film for IB wt $\%>0$. The electrical conductivity is high at RT and decreases with increasing temperature for the films with IB wt $\%>0$ because of a decrease in carrier mobility (c.f. Figure $5 b$ ). The metallic behavior of the films indicates that the charge transport of all the films with IB wt $\%>0$ is dominated by the highly conducting $\beta-\mathrm{Cu}_{2-\delta}$ Se phase connecting the BST grains in the composite. To estimate the thermal conductivity of the printed films, cylindrical pellets were made by cold pressing using the TE inks followed by sintering. The thermal conductivities of the pellets were measured by the laser flash method.

It should be noted that because of the difficulties in measuring the in-plane thermal conductivities of the printed films using a thin-film analyzer setup, we adopted the abovementioned procedure to estimate the $\kappa$ values. Moreover, the density of the pellets is much higher than that for printed films because of the expulsion of solvent and resin during pellet preparation. Therefore, it is assumed that the thermal conductivity of the printed films would not be higher than that of the pellets. The $\kappa$ for all the compositions decreases slightly with increasing temperature and is estimated to be lower compared to that of their bulk values. The low $\kappa$ value is mainly due to the low density of the samples $(<50 \%$ of theoretical density) and the reduced heat transfer in the 

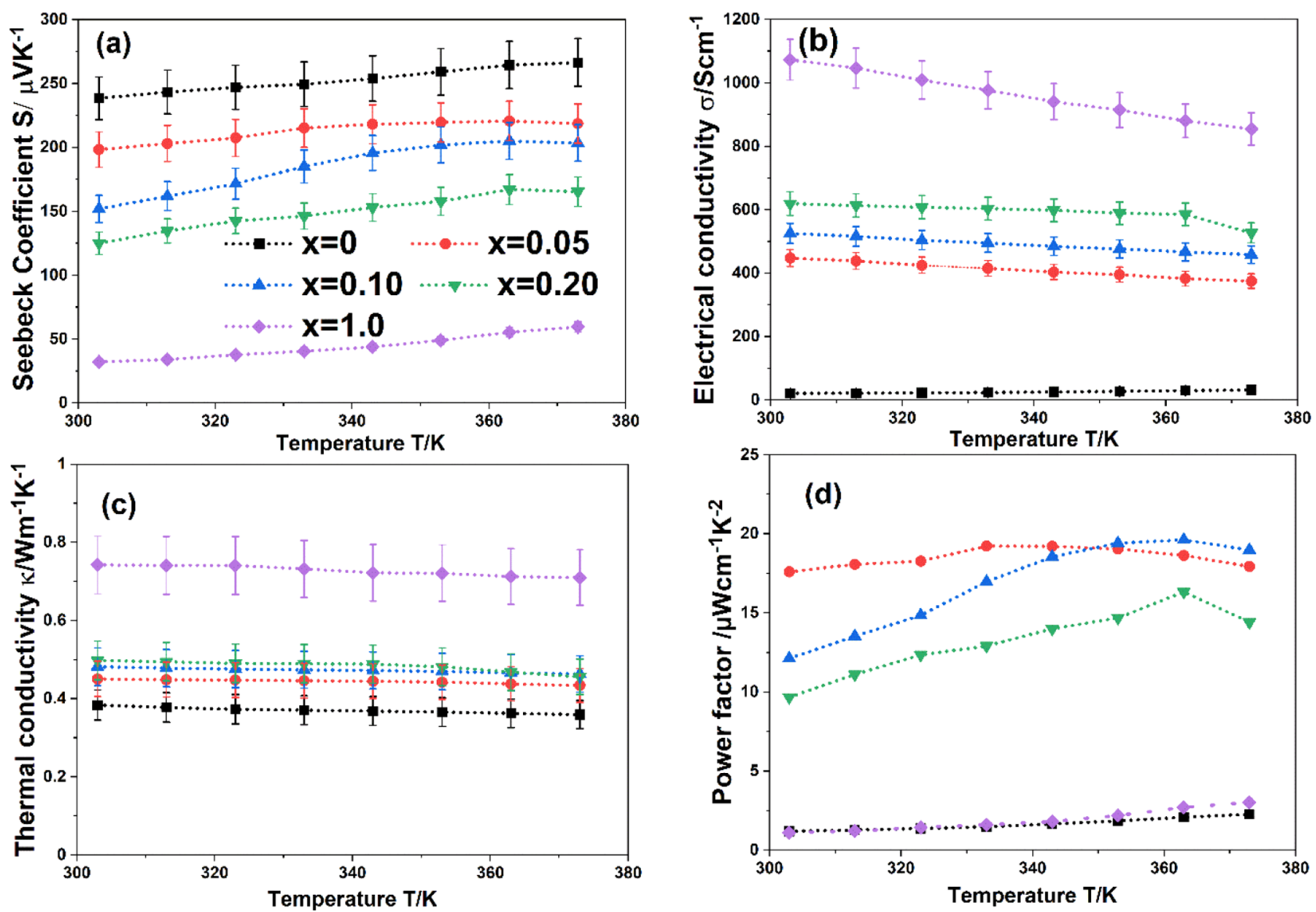

Figure 5. Variation of TE parameters $S(\mathrm{a}), \sigma(\mathrm{b})$, and the estimated $\kappa$ of the printed materials (c) with temperatures from 300 to $400 \mathrm{~K}$ of the films for $x=0$ to 1.0. Temperature-dependent power factor $S^{2} \sigma$ for all films is shown in (d). The films with IB wt $\%$ of 5 and 10 exhibit high $S^{2} \sigma$ reaching a maximum value for the power factor of $\sim 20 \mu \mathrm{W} \mathrm{cm}{ }^{-1} \mathrm{~K}^{-2}$ at $360 \mathrm{~K}$.
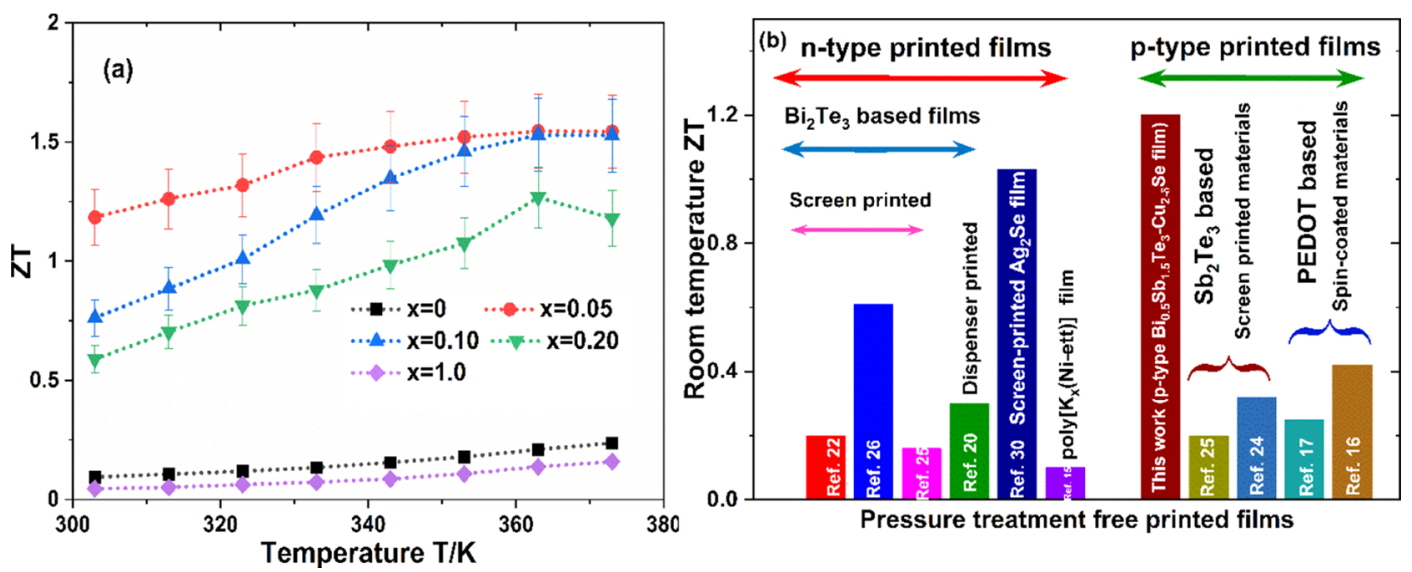

Figure 6. Estimated temperature-dependent figure-of-merit for all the films for $x=$ to 1.0 . The highest $Z T$ of $>1.5$ is estimated by the films with $x=$ 0.05 and 0.10 at $T>350 \mathrm{~K}$ (a). This is the highest $Z T$ value achieved for a pressure-treatment-free printed film (b).

presence of remnant organic binders and additives. The lowest $\kappa$ of $\sim 0.30 \mathrm{~W} \mathrm{~m}^{-1} \mathrm{~K}^{-1}$ is found for $x=0$ and increases with increasing IB content at $\mathrm{RT}$, reaching a maximum value of $\sim 0.75 \mathrm{~W} \mathrm{~m}^{-1} \mathrm{~K}^{-1}$ for the film with $x=0$ (c.f. Figure $5 \mathrm{c}$ ). The lattice contribution to the $\kappa$ is dominant for the pristine film with a negligible contribution from carrier transport, and a more prominent electronic contribution to $\kappa$ is found for higher IB contents. The temperature-dependent power factor, $S^{2} \sigma$, for all the films is calculated using $S$ and $\sigma$ and is shown in Figure $5 \mathrm{~d}$. The highest power factor value of $\sim 18 \mu \mathrm{W} \mathrm{cm}-1$
$\mathrm{K}^{-2}$ at RT is found for $x=0.05$, and a maximum value of $\sim 20$ $\mu \mathrm{W} \mathrm{cm} \mathrm{c}^{-1} \mathrm{~K}^{-2}$ is reached at $360 \mathrm{~K}$ for $x=0.10$. Although the power factor value is lower than that for the bulk Bi-Sb-Te alloy, the value is significantly high for a printed material. The lower value of the power factor is mainly due to the lower electrical conductivity of the printed films compared to that of the bulk. The temperature-dependent figures-of-merit $Z T$ is estimated from the power factors and the thermal conductivity values for all the films shown in Figure 6. The $Z T$ is found to be increased with temperature for all the films. A high $Z T$ of 

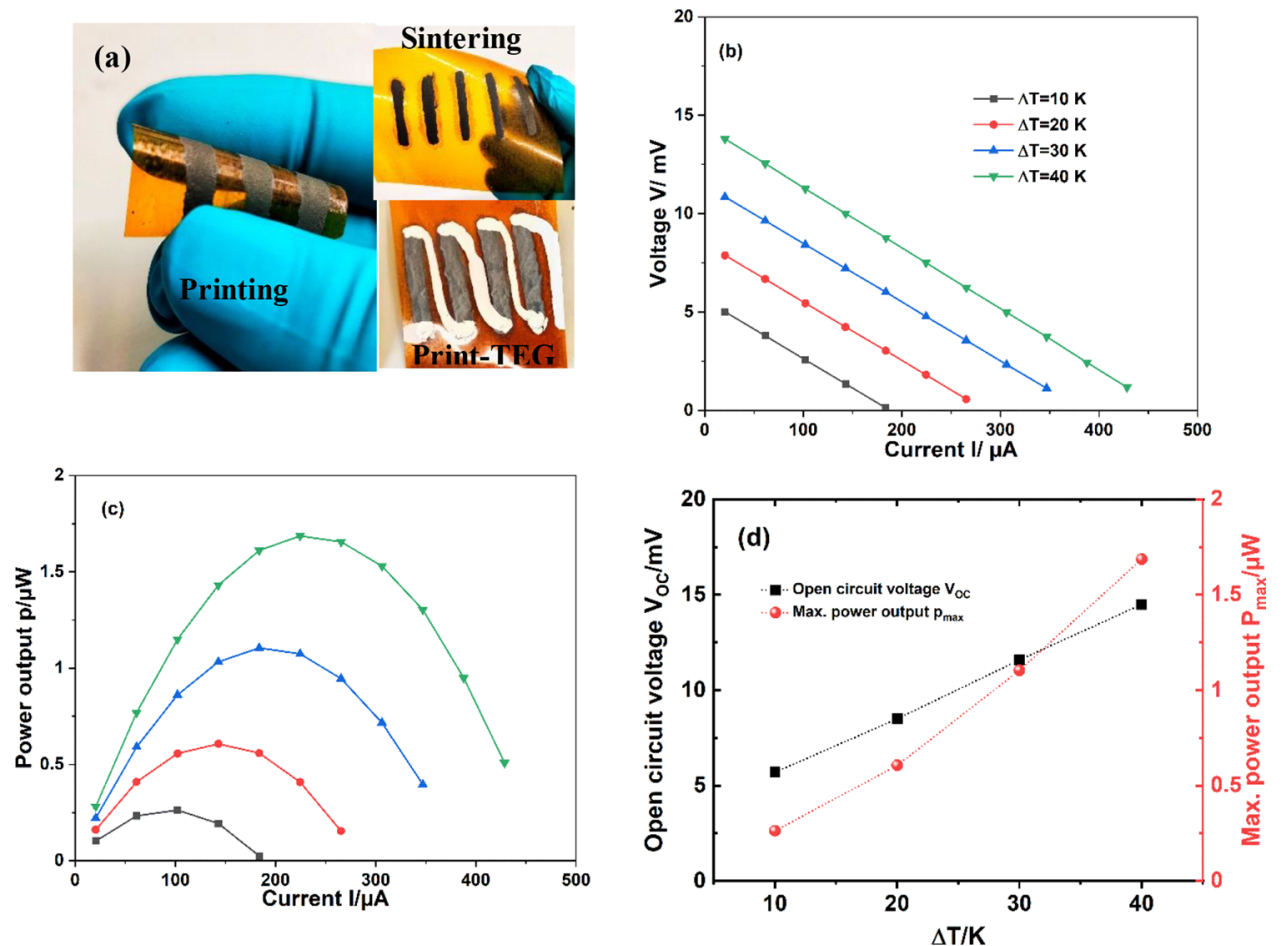

Figure 7. Printed BST-based film and print-TEG (a). The variation of output voltage (b) and output power (c) of the print-TEG with the current while operating at different $\Delta T$ s. The $\Delta T$-dependent open-circuit voltage $\left(V_{\mathrm{OC}}\right)$ and maximum power output $\left(P_{\max }\right)$ of the print-TEG are shown in (d), respectively.

$\sim 1.2$ at RT is appraised in the film with $x=0.05$, which increases with temperature. An impressive maximum $Z T$ of $>1.5$ is estimated with the films with $x=0.05$ and $x=0.10$ at a temperature $T>350 \mathrm{~K}$. This is the highest reported figure-ofmerit value for a printed TE film without any high-pressure post-treatment to the best of our knowledge.

3.3. Performance of the Printed TEG. First, four films with a dimension of $\sim 4 \mathrm{~cm} \times 0.5 \mathrm{~cm} \times 0.015 \mathrm{~cm}$ are printed on a Kapton substrate using the prepared printable ink containing 10 wt \% of IB for p-type legs. The Kapton substrate was rubbed using emery paper to make the surface rough for better adhesion of the films. Subsequently, the films were sintered at $703 \mathrm{~K}$ for $10 \mathrm{~min}$ followed by connecting the films electrically in series and thermally in parallel by silver paste to fabricate a TE generator (print-TEG). The print-TEG was characterized by the maximum power point tracking method varying the device current from 0 to $500 \mu \mathrm{A}$ and the temperature difference $\Delta T$ from 10 to $40 \mathrm{~K}$. The output voltage of the print-TEG was measured using a KEITHLEY source measuring Unit 2601B. Hereby the one side of the print-TEG was thermally coupled to the hot unit of a measuring setup, and another side of the device was exposed at ambient temperature (cf. Figure S5). The working principle of the TEG measurement setup is given in Supplementary Note 2 of the previous report. ${ }^{33}$ The performance of the printTEG is shown in Figure 7. An open-circuit voltage of $14 \mathrm{mV}$ and a maximum power output of $1.7 \mu \mathrm{W}$ are achieved for $\Delta T$ $=40 \mathrm{~K}$. As the estimated cross-sectional area of the print-TEG through which heat is conducted from the hot to cold side $\sim 0.04 \mathrm{~cm}^{2}$, the maximum power output density is calculated to be $\sim 42.5 \mu \mathrm{Wcm}^{-2}$.

\section{DISCUSSION}

The major challenge in developing a high-performance printed TE material using particles made from conventional TE materials is to preserve its high electrical conductivity. The detrimental effects on the $S$ and $\kappa$ are not significant, while the $\sigma$ is reduced critically in a printed material because of an increase in interfacial resistance. The interfacial resistance is increased at the grain boundaries because of insulating organic binders, additives, and changes in the chemical structure of the surfaces of the TE particles by reacting with solvent molecules (e.g., oxidation). To overcome these challenges, the grain boundary interfaces of BST particles are modified using a high electrical/low thermal conductivity $\beta-\mathrm{Cu}_{2-\delta} \mathrm{Se}$ phase. The bandgap of the pristine BST film is calculated using the Seebeck coefficient via the following expression; ${ }^{37} E_{\mathrm{g}}=$ $2 q S_{\max } T_{\max }$ ( $q$ is the elementary charge and $S_{\max }$ and $T_{\max }$ are the maximum Seebeck coefficient and the temperature where $S$ is maximum). Generally, the $S_{\max }$ for the $\mathrm{Bi}_{0.5} \mathrm{Sb}_{1.5} \mathrm{Te}_{3}$ is reported to be around $400 \mathrm{~K}, T_{\max }{ }^{38}$ Also, the $S$ value for the pristine $\mathrm{Bi}_{0.5} \mathrm{Sb}_{1.5} \mathrm{Te}_{3}$ film remains almost unchanged between 360 and $380 \mathrm{~K}$ in this work. Hence, considering $S_{\max }$ to be at $380 \mathrm{~K}$, the bandgap of the pristine BST film is estimated to be $\sim 0.18 \mathrm{eV}$. The cubic $\beta-\mathrm{Cu}_{2-\delta} \mathrm{Se}$ phase with a low bandgap of $\sim 0.1 \mathrm{eV}$ is formed in between the BST grains with a higher bandgap during sintering through dissociative adsorption of Se by $\mathrm{Cu}$. As a result, interruption of charge-carrier transport at the grain boundaries is significantly reduced compared to conventional printed materials with high interfacial resistance. Furthermore, the $\beta-\mathrm{Cu}_{2-\delta} \mathrm{Se}$ phase has a higher carrier concentration $p_{\mathrm{H}}$ and electrical conductivity $\sigma$ than BST with a low thermal conductivity $\kappa$ value and facilitates a modulationdoped-like system. Therefore, the overall carrier concentration and $\sigma$ of the BST/IB composite films are increased with 
Table 2. Maximum Power Output per Thermocouple for Currently Reported Printed TEGs

\begin{tabular}{|c|c|c|c|c|}
\hline TE legs & printing methods & max. power output per thermocouple $/ \mathrm{nW}$ & $\Delta T$ & references \\
\hline n-type: n-doped CNT and p-type: p-doped CNT & $3 \mathrm{D}$ printing & 28.3 & $30 \mathrm{~K}$ & 39 \\
\hline n-type: $(\text { PEDOT })_{0.03} \mathrm{~V}_{2} \mathrm{O}_{5}$ and p-type: PEDOT:PSS & inkjet-printing & 0.087 & $20 \mathrm{~K}$ & 40 \\
\hline p-type: $\mathrm{Bi}_{2} \mathrm{Te}_{2.7} \mathrm{Se}_{0.3}$ and n-type: $\mathrm{Ag}$ & $3 \mathrm{D}$ aerosol jet printing & 35 & $50 \mathrm{~K}$ & 41 \\
\hline $\mathrm{Sb}_{2} \mathrm{Te}_{3}$ and $\mathrm{Bi}_{1.8} \mathrm{Te}_{3.2}$ & screen printing & 50 & $20 \mathrm{~K}$ & 42 \\
\hline n-type: $\mathrm{Bi}_{2} \mathrm{Te}_{3}$ and p-type: $\mathrm{Bi}_{0.5} \mathrm{Sb}_{1.5} \mathrm{Te}_{3}$ & inkjet-printing & 25.4 & $32.5 \mathrm{~K}$ & 23 \\
\hline n-type: $\mathrm{Ag}_{2} \mathrm{Se}$ and p-type: PEDOT & painted on 3D scaffolds & 75 & $70 \mathrm{~K}$ & 12 \\
\hline n-type: $\mathrm{Ag}$ and p-type: $\mathrm{Bi}_{0.5} \mathrm{Sb}_{1.5} \mathrm{Te}_{3}$ & doctor blading & 422 & $45 \mathrm{~K}$ & this work \\
\hline
\end{tabular}

increasing $\beta-\mathrm{Cu}_{2-\delta} \mathrm{Se}$ contents. As a result, even though the Seebeck coefficient $S$ also decreases, the power factor and $Z T$ are increased significantly because of the large enhancement of $\sigma$. Consequently, the uni-leg print-TEG fabricated using the ptype BST-IB material has exhibited a promising power output of $\sim 422 \mathrm{nW} /$ leg. A comparison of power outputs from different printed TEGs is given in Table 2. However, the estimated Seebeck coefficient per leg using eq 1 is found to be lower than the material Seebeck coefficient.

$$
\left(S_{\mathrm{p}}-S_{\mathrm{n}}\right)=\frac{V_{\mathrm{OC}}}{(\Delta T \times N)}
$$

$S_{\mathrm{p}}$ is the Seebeck coefficient of the p-type BST-IB film, $S_{\mathrm{n}}$ is the Seebeck coefficient of the silver, and $N$ is the number of legs. The $\left(S_{\mathrm{p}}-S_{\mathrm{n}}\right)$ is determined to be $143 \mu \mathrm{V} \mathrm{K}-1$ for $\Delta T=$ $10 \mathrm{~K}$, and it decreases at higher $\Delta T$ s (c.f. Figure S6). The reduced thermal voltage output because of the thermal contact resistance and Joule heating might be the reason behind the lower Seebeck coefficient per leg. Also, the actual temperature difference $\Delta T_{\text {actual }}$ between the hot and cold ends of the TEG is lower compared to its set value $\Delta T$ during device operation.

The resultant power output of a TEG depends on the total number of thermocouples $(N)$, the device internal resistance $R_{\mathrm{I}}$, the temperature difference $\Delta T$ between the hot and cold sides, and Seebeck coefficient values of the TE legs. Hence, the device power output can be expressed as

$$
\begin{aligned}
& P=\frac{V_{\mathrm{OC}}^{2}}{\left(R_{\mathrm{L}}+R_{\mathrm{I}}\right)^{2}} R_{L}=\frac{\left[\left(S_{\mathrm{p}}-S_{\mathrm{n}}\right) \times(\Delta T \times N)\right]^{2} R_{\mathrm{L}}}{\left(R_{\mathrm{L}}+R_{\mathrm{I}}\right)^{2}} \\
& P_{\max }=\frac{\left[\left(S_{\mathrm{p}}-S_{\mathrm{n}}\right) \times(\Delta T \times N)\right]^{2}}{4 R_{\mathrm{I}}} \text { for } R_{\mathrm{L}}=R_{\mathrm{I}}
\end{aligned}
$$

where $R_{\mathrm{I}}$ is the total internal resistance of the TEG, $R_{\mathrm{L}}$ is the load resistance, $S_{\mathrm{n}}$ and $S_{\mathrm{p}}$ are the Seebeck coefficients of the nand p-type TE legs, respectively, and $P_{\max }$ is the maximum power output. If the internal resistance of a thermocouple is $R_{\mathrm{i}}$, then $R_{\mathrm{I}}=N R_{\mathrm{i}}$. The $R_{\mathrm{i}}$ depends on the electrical conductivity, cross-sectional area, and length $\sigma_{\mathrm{p}}, A_{\mathrm{p}} L_{\mathrm{p}}$ and $\sigma_{\mathrm{n}}, A_{\mathrm{n}} L_{\mathrm{n}}$ for the p-type and the n-type legs, respectively.

$$
R_{\mathrm{i}}=\left[\left(\sigma_{\mathrm{n}} \frac{A_{\mathrm{n}}}{L_{\mathrm{n}}}\right)^{-1}+\left(\sigma_{\mathrm{p}} \frac{A_{\mathrm{p}}}{L_{\mathrm{p}}}\right)^{-1}\right]
$$

Hence, the performance of the print-TEG can be improved by adjusting the dimension and the number of the TE legs. An estimated power output of $>100 \mu \mathrm{W}$ could be achieved by the print-TEG increasing cross-section area by two times and the number $(N=100)$ of the TE legs.

\section{CONCLUSIONS}

The main challenge in printed thermoelectrics is to preserve high electrical conductivity after printing a conventional TE material with high performance to overcome the high interfacial resistance. Furthermore, insulating organic binders, additives, and chemical modification of the grain surfaces of the TE particles increase the interfacial resistance. In our work, the grain interfaces of BST particles in printed films have been modified using a low bandgap, high $\sigma$, and low- $\kappa$ material, $\beta$ $\mathrm{Cu}_{2-\delta}$ Se. The $\beta-\mathrm{Cu}_{2-\delta}$ Se phase is formed between BST grains through a mild sintering process on a Kapton substrate creating a highly conducting path. As a result, a high electrical conductivity $\sigma$ together with low $\kappa$ and moderately high $\mathrm{S}$ is attained in the printed films $(1-x) \mathrm{BST}-(x)$ IB with $0<x<1$. A very high TE power factor of $\sim 17.5 \mu \mathrm{W} \mathrm{cm} \mathrm{cm}^{-1} \mathrm{~K}^{-2}$ with a record-high $Z T$ of $\sim 1.2$ is achieved in the printed film with $x=$ 0.05 at RT. A printed TE generator with four thermocouples has been fabricated using the film with $x=0.05$ material for $\mathrm{p}$ type TE legs and silver as n-type TE legs. The print-TEG exhibited an open-circuit voltage of $14 \mathrm{mV}$ with a maximum power output of $1.7 \mu \mathrm{W}$ for $\Delta T=40 \mathrm{~K}$.

\section{ASSOCIATED CONTENT}

\section{S1 Supporting Information}

The Supporting Information is available free of charge at https://pubs.acs.org/doi/10.1021/acsami.1c13526.

The temperature-dependent XRD pattern of the TE ink for $x=1$ and the crystallographic structure of the cubic $\beta-\mathrm{Cu}_{2-\delta} \mathrm{Se}$ phase (Figure $\mathrm{S} 1$ ), morphological and elemental analysis of the printed films (Figures S2S4), schematic diagram of the TEG measuring setup (Figure S5), and Seebeck coefficient per thermocouple for different $\Delta T$ s (Figure S6) (PDF)

A video demonstration of the printed film (MP4)

\section{AUTHOR INFORMATION}

\section{Corresponding Authors}

Md Mofasser Mallick - Light Technology Institute, Karlsruhe Institute of Technology, 76131 Karlsruhe, Germany; () orcid.org/0000-0003-2105-6153; Email: mofasser.mallick@kit.edu

Uli Lemmer - Light Technology Institute, Karlsruhe Institute of Technology, 76131 Karlsruhe, Germany; Institute of Microstructure Technology Karlsruhe Institute of Technology, 76344 Eggenstein-Leopoldshafen, Germany; (1) orcid.org/ 0000-0001-9892-329X; Email: uli.lemmer@kit.edu

\section{Authors}

Leonard Franke - Light Technology Institute, Karlsruhe Institute of Technology, 76131 Karlsruhe, Germany 
Andres Georg Rösch - Light Technology Institute, Karlsruhe Institute of Technology, 76131 Karlsruhe, Germany

Sarfraz Ahmad - Institute for Applied Materials Karlsruhe Institute of Technology, 76344 Eggenstein-Leopoldshafen, Germany

Holger Geßwein - Institute for Applied Materials Karlsruhe Institute of Technology, 76344 Eggenstein-Leopoldshafen, Germany

Yolita M. Eggeler - Laboratory for electron microscopy, Karlsruhe Institute of Technology, 76131 Karlsruhe, Germany

Magnus Rohde - Institute for Applied Materials Karlsruhe Institute of Technology, 76344 Eggenstein-Leopoldshafen, Germany

Complete contact information is available at: https://pubs.acs.org/10.1021/acsami.1c13526

\section{Notes}

The authors declare no competing financial interest.

The CIF data for the $\beta-\mathrm{Cu}_{2-\delta} \mathrm{Se}$ have been assigned to the following deposition numbers, CCDC 2084678.

\section{ACKNOWLEDGMENTS}

The authors wish to acknowledge the Deutsche Forschungsgemeinschaft (DFG, German Research Foundation) under Germany's Excellence Strategy via the Excellence Cluster 3D Matter Made to Order (EXC-2082/1-390761711) for financial support. The authors wish to acknowledge funding by the Ministry of Science, Research, and Arts of the state of Baden Württemberg through the MERAGEM graduate school. This project has received funding from the European Union's Horizon 2020 research and innovation program under grant agreement No. 814945 - SolBio-Rev. The German Federal Environmental Foundation (Deutsche Bundesstiftung Umwelt-DBU) through the DBU Ph.D. scholarship program also supported this work.

\section{REFERENCES}

(1) Prilepo, Y. P.; Pustovalov, A. A.; Sinyavskiy, V. V.; Sudak, N. M.; Yatsenko, O. B. Problems of Designing Radioisotope Thermoelectric Power Generators with a Service Life of Decades for Use in Outer Space Exploration Vehicles. Therm. Eng. 2012, 59, 981-983.

(2) Snyder, G. J.; Toberer, E. S. Complex Thermoelectric Materials. Nat. Mater. 2008, 7, 105-114.

(3) Twaha, S.; Zhu, J.; Yan, Y.; Li, B. A Comprehensive Review of Thermoelectric Technology: Materials, Applications, Modelling and Performance Improvement. Renewable Sustainable Energy Rev. 2016, 65, 698-726.

(4) Wei, J.; Yang, L.; Ma, Z.; Song, P.; Zhang, M.; Ma, J.; Yang, F.; Wang, X. Review of Current High-ZT Thermoelectric Materials. J. Mater. Sci. 2020, 55, 12642-12704.

(5) Sharma, A.; Lee, J. H.; Kim, K. H.; Jung, J. P. Recent Advances in Thermoelectric Power Generation Technology. J. Microelectron. Packag. Soc. 2017, 24, 9-16.

(6) Yin, Y.; Baskaran, K.; Tiwari, A. A Review of Strategies for Developing Promising Thermoelectric Materials by Controlling Thermal Conduction. Phys. Status Solidi Appl. Mater. Sci. 2019, 216, No. 1800904.

(7) Ma, Z.; Wei, J.; Song, P.; Zhang, M.; Yang, L.; Ma, J.; Liu, W.; Yang, F.; Wang, X. Review of Experimental Approaches for Improving ZT of Thermoelectric Materials. Mater. Sci. Semicond. Process. 2021, 121, No. 105303.

(8) Mallick, M. M.; Vitta, S. Realizing High Figure-of-Merit in $\mathrm{Cu}_{2} \mathrm{Te}$ Using a Combination of Doping, Hierarchical Structure, and Simple Processing. J. Appl. Phys. 2017, 122, No. 024903.
(9) Aswal, D. K.; Basu, R.; Singh, A. Key Issues in Development of Thermoelectric Power Generators: High Figure-of-Merit Materials and Their Highly Conducting Interfaces with Metallic Interconnects. Energy Convers. Manage. 2016, 114, 50-67.

(10) Du, Y.; Chen, J.; Meng, Q.; Dou, Y.; Xu, J.; Shen, S. Z. Thermoelectric Materials and Devices Fabricated by Additive Manufacturing. Vacuum 2020, 178, No. 109384.

(11) Kim, J.; Kumar, R.; Bandodkar, A. J.; Wang, J. Advanced Materials for Printed Wearable Electrochemical Devices: A Review. Adv. Electron. Mater. 2017, 3, No. 1600260.

(12) Mallick, M.; Franke, L.; Rösch, A. G.; Lemmer, U. ShapeVersatile 3D Thermoelectric Generators by Additive Manufacturing. ACS Energy Lett. 2021, 6, 85-91.

(13) Zhang, Y.; Heo, Y. J.; Park, M.; Park, S. J. Recent Advances in Organic Thermoelectric Materials: Principle Mechanisms and Emerging Carbon-Based Green Energy Materials. Polymers 2019, $11,167$.

(14) Lindorf, M.; Mazzio, K. A.; Pflaum, J.; Nielsch, K.; Brütting, W.; Albrecht, M. Organic-Based Thermoelectrics. J. Mater. Chem. A 2020, 8, 7495-7507.

(15) Sun, Y.; Sheng, P.; Di, C.; Jiao, F.; Xu, W.; Qiu, D.; Zhu, D. Organic Thermoelectric Materials and Devices Based on P- and nType Poly (Metal 1, 1,2, 2-Ethenetetrathiolate)S. Adv. Mater. 2012, 24, 932-937.

(16) Kim, G. H.; Shao, L.; Zhang, K.; Pipe, K. P. Engineered Doping of Organic Semiconductors for Enhanced Thermoelectric Efficiency. Nat. Mater. 2013, 12, 719-723.

(17) Bubnova, O.; Khan, Z. U.; Malti, A.; Braun, S.; Fahlman, M.; Berggren, M.; Crispin, X. Optimization of the Thermoelectric Figure of Merit in the Conducting Polymer Poly(3,4-Ethylenedioxythiophene). Nat. Mater. 2011, 10, 429-433.

(18) Pei, J.; Cai, B.; Zhuang, H. L.; Li, J. F. Bi2Te3-Based Applied Thermoelectric Materials: Research Advances and New Challenges. Natl. Sci. Rev. 2020, 7, 1856-1858.

(19) Witting, I. T.; Chasapis, T. C.; Ricci, F.; Peters, M.; Heinz, N. A.; Hautier, G.; Snyder, G. J. The Thermoelectric Properties of Bismuth Telluride. Adv. Electron. Mater. 2019, 5, No. 1800904.

(20) Wu, K.; Yan, Y.; Zhang, J.; Mao, Y.; Xie, H.; Yang, J.; Zhang, Q.; Uher, C.; Tang, X. Preparation of N-Type Bi2Te3 Thermoelectric Materials by Non-Contact Dispenser Printing Combined with Selective Laser Melting. Phys. Status Solidi RRL 2017, 11, No. 1700067.

(21) Varghese, T.; Dun, C.; Kempf, N.; Saeidi-Javash, M.; Karthik, C.; Richardson, J.; Hollar, C.; Estrada, D.; Zhang, Y. Flexible Thermoelectric Devices of Ultrahigh Power Factor by Scalable Printing and Interface Engineering. Adv. Funct. Mater. 2020, 30, No. 1905796

(22) Feng, J.; Zhu, W.; Deng, Y.; Song, Q.; Zhang, Q. Enhanced Antioxidation and Thermoelectric Properties of the Flexible ScreenPrinted Bi2Te3 Films through Interface Modification. ACS Appl. Energy Mater. 2019, 2, 2828-2836.

(23) Chen, B.; Kruse, M.; Xu, B.; Tutika, R.; Zheng, W.; Bartlett, M. D.; Wu, Y.; Claussen, J. C. Flexible Thermoelectric Generators with Inkjet-Printed Bismuth Telluride Nanowires and Liquid Metal Contacts. Nanoscale 2019, 11, 5222-5230.

(24) Kim, S. J.; We, J. H.; Kim, J. S.; Kim, G. S.; Cho, B. J. Thermoelectric Properties of P-Type $\mathrm{Sb}_{2} \mathrm{Te}_{3}$ Thick Film Processed by a Screen-Printing Technique and a Subsequent Annealing Process. J. Alloys Compd. 2014, 582, 177-180.

(25) We, J. H.; Kim, S. J.; Cho, B. J. Hybrid Composite of ScreenPrinted Inorganic Thermoelectric Film and Organic Conducting Polymer for Flexible Thermoelectric Power Generator. Energy 2014, 73, 506-512.

(26) We, J. H.; Kim, S. J.; Kim, G. S.; Cho, B. J. Improvement of Thermoelectric Properties of Screen-Printed $\mathrm{Bi}_{2} \mathrm{Te}_{3}$ Thick Film by Optimization of the Annealing Process. J. Alloys Compd. 2013, 552, 107-110.

(27) Kim, F.; Kwon, B.; Eom, Y.; Lee, J. E.; Park, S.; Jo, S.; Park, S. H.; Kim, B. S.; Im, H. J.; Lee, M. H.; Min, T. S.; Kim, K. T.; Chae, H. 
G.; King, W. P.; Son, J. S. 3D Printing of Shape-Conformable Thermoelectric Materials Using All-Inorganic $\mathrm{Bi}_{2} \mathrm{Te}_{3}$-Based Inks. Nat. Energy 2018, 3, 301-309.

(28) Park, S. H.; Jo, S.; Kwon, B.; Kim, F.; Ban, H. W.; Lee, J. E.; Gu, D. H.; Lee, S. H.; Hwang, Y.; Kim, J. S.; Hyun, D. B.; Lee, S.; Choi, K. J.; Jo, W.; Son, J. S. High-Performance Shape-Engineerable Thermoelectric Painting. Nat. Commun. 2016, 7, 13403.

(29) Zhao, X.; Han, W.; Jiang, Y.; Zhao, C.; Ji, X.; Kong, F.; Xu, W.; Zhang, X. A Honeycomb-like Paper-Based Thermoelectric Generator Based on a Bi2Te3/Bacterial Cellulose Nanofiber Coating. Nanoscale 2019, 11, 17725-17735.

(30) Mallick, M. M.; Rösch, A. G.; Franke, L.; Gall, A.; Ahmad, S.; Geßwein, H.; Mazilkin, A.; Kübel, C.; Lemmer, U. New Frontier in Printed Thermoelectrics: Formation of $\beta-\mathrm{Ag}_{2} \mathrm{Se}$ through Thermally Stimulated Dissociative Adsorption Leads to High: ZT. J. Mater. Chem. A 2020, 8, 16366-16375.

(31) Mallick, M. M.; Rösch, A. G.; Franke, L.; Ahmed, S.; Gall, A.; Geßwein, H.; Aghassi, J.; Lemmer, U. High-Performance Ag-Se-Based n-Type Printed Thermoelectric Materials for High Power Density Folded Generators. ACS Appl. Mater. Interfaces 2020, 12, 1965519663.

(32) Mallick, M. M.; Vitta, S. Enhancing the Thermoelectric Performance of a P-Type Half-Heusler Alloy, HfCoSb by Incorporation of a Band-Matched Chalcogenide, $\mathrm{Cu}_{2}$ Te. J. Mater. Chem. A 2018, 6, 14709-14716.

(33) Rösch, A. G.; Gall, A.; Aslan, S.; Hecht, M.; Franke, L.; Mallick, M. M.; Penth, L.; Bahro, D.; Friderich, D.; Lemmer, U. Fully Printed Origami Thermoelectric Generators for Energy-Harvesting. npj Flex Electron. 2021, 5, 1.

(34) Scimeca, M. R.; Yang, F.; Zaia, E.; Chen, N.; Zhao, P.; Gordon, M. P.; Forster, J. D.; Liu, Y. S.; Guo, J.; Urban, J. J.; Sahu, A. Rapid Stoichiometry Control in $\mathrm{Cu}_{2} \mathrm{Se}$ Thin Films for Room-Temperature Power Factor Improvement. ACS Appl. Energy Mater. 2019, 2, 15171525.

(35) Holcomb, D. F.; Iwasawa, M.; Roberts, F. D. K. Clustering of Randomly Placed Spheres. Biometrika 1972, 59, 207-209.

(36) Holcomb, D. F.; Rehr, J. J. Percolation in Heavily Doped Semiconductors. Phys. Rev. 1969, 183, 773-776.

(37) Zhang, Q.; Fang, T.; Liu, F.; Li, A.; Wu, Y.; Zhu, T.; Zhao, X. Tuning Optimum Temperature Range of $\mathrm{Bi}_{2} \mathrm{Te}_{3}$-Based Thermoelectric Materials by Defect Engineering. Chem. - Asian J. 2020, 15, 2775-2792.

(38) Deng, R.; Su, X.; Zheng, Z.; Liu, W.; Yan, Y.; Zhang, Q.; Dravid, V. P.; Uher, C.; Kanatzidis, M. G.; Tang, X. Thermal Conductivity in $\mathrm{Bi}_{0.5} \mathrm{Sb}_{1.5} \mathrm{Te}_{3+\mathrm{x}}$ and the Role of Dense Dislocation Arrays at Grain Boundaries. Sci. Adv. 2018, 6, 7358-7365.

(39) Park, K. T.; Choi, J.; Lee, B.; Ko, Y.; Jo, K.; Lee, Y. M.; Lim, J. A.; Park, C. R.; Kim, H. High-Performance Thermoelectric Bracelet Based on Carbon Nanotube Ink Printed Directly onto a Flexible Cable. J. Mater. Chem. A 2018, 6, 19727-19734.

(40) Ferhat, S.; Domain, C.; Vidal, J.; Noël, D.; Ratier, B.; Lucas, B. Organic Thermoelectric Devices Based on a Stable N-Type Nanocomposite Printed on Paper. Sustain. Energy Fuels 2018, 2, 199-208.

(41) Saeidi-Javash, M.; Kuang, W.; Dun, C.; Zhang, Y. 3D Conformal Printing and Photonic Sintering of High-Performance Flexible Thermoelectric Films Using 2D Nanoplates. Adv. Funct. Mater. 2019, 29, No. 1901930.

(42) Cao, Z.; Koukharenko, E.; Tudor, M. J.; Torah, R. N.; Beeby, S. P. Flexible Screen Printed Thermoelectric Generator with Enhanced Processes and Materials. Sens. Actuators, A 2016, 238, 196-206. 